\title{
Strates
}

STRATES Matériaux pour la recherche en sciences sociales

$10 \mid 2001$

Villageois et citadins de Grèce

\section{Usage des langues minoritaires dans les départements de Florina et d'Aridea (Macédoine)}

\section{Riki Van Boeschoten}

\section{(2) OpenEdition \\ 12 Journals}

Édition électronique

URL : http://journals.openedition.org/strates/381

ISSN : $1777-5442$

Éditeur

Laboratoire Ladyss

Édition imprimée

Date de publication : 1 mai 2001

ISSN : 0768-8067

\section{Référence électronique}

Riki Van Boeschoten, « Usage des langues minoritaires dans les départements de Florina et d'Aridea (Macédoine) », Strates [En ligne], 10 | 2001, mis en ligne le 11 janvier 2005, consulté le 07 septembre 2020. URL : http://journals.openedition.org/strates/381

Ce document a été généré automatiquement le 7 septembre 2020.

Tous droits réservés 


\title{
Usage des langues minoritaires dans les départements de Florina et d'Aridea (Macédoine)
}

\author{
Riki Van Boeschoten
}

1 Ce document présente les principaux résultats d'une enquête de terrain effectuée en septembre 1993 et financée par la Commission européenne ${ }^{1}$. L'objectif de cette enquête était d'analyser l'usage des langues minoritaires dans une région de la Grèce du nord qui se présente comme une mosaïque linguistique. Dans cette région formée des départements de Florina et d'Aridea, en dehors de la langue dominante (le grec), sont encore en usage les langues minoritaires suivantes : le macédonien, le valaque (proche du roumain), l'arvanitika (proche de l'albanais), le turc, le rom et le dialecte pontique (un dialecte grec utilisé par les anciens habitants de la mer Noire ou Pontos). Notre équipe était composée de deux anthropologues néerlandaises, Riki Van Boeschoten et Helleen van der Minne. Nous avons visité en tout 72 des 139 villages de la région.

2 Notre recherche a été conçue comme une enquête intégrant les phénomènes linguistiques à tous les aspects du contexte socio-économique. Dans chaque village, nous avons pris contact avec le maire ou le secrétaire du village puis nous l'avons interrogé à l'aide d'un questionnaire. Si aucun des deux n'était présent dans le village, nous avons pris contact avec d'autres villageois. Les villageois inclus dans la recherche représentent environ $65 \%$ du groupe slavophone, $90 \%$ du groupe parlant le valaque et $100 \%$ du groupe parlant l'arvanitika, sur l'ensemble de la population des deux départements. La distribution des groupes linguistiques est indiquée dans les tableaux 1 et 2 .

Distribution des groupes linguistiques

Tableau 1 - Population rurale du département de Florina

\begin{tabular}{|l|l|l|}
\hline $\begin{array}{l}\text { Groupes } \\
\text { linguistiques }\end{array}$ & $\begin{array}{l}\text { Nombre } \\
\text { de villages }\end{array}$ & Habitants \\
\hline
\end{tabular}




\begin{tabular}{|l|l|l|l|}
\hline Slavophones & 43 & 15228 & 42 \\
\hline \hline Arvanites & 3 & 2114 & 6 \\
\hline \hline Valaques & 6 & 789 & 2 \\
\hline \hline Réfugiés grecs & 13 & 5554 & 15 \\
\hline \hline Mixtes & 29 & 12527 & 35 \\
\hline \hline TOTAL & 94 & 36212 & 100 \\
\hline
\end{tabular}

Tableau 2 - Population rurale du département d'Aridea

\begin{tabular}{|l|l|l|l|}
\hline $\begin{array}{l}\text { Groupes } \\
\text { linguistiques }\end{array}$ & $\begin{array}{l}\text { Nombre } \\
\text { de villages }\end{array}$ & Habitants & $\%$ \\
\hline \hline Slavophones & 10 & 5502 & 22 \\
\hline \hline Valaques & 3 & 1181 & 4 \\
\hline \hline Réfugiés grecs & 15 & 5515 & 22 \\
\hline \hline Mixtes & 17 & 12527 & 50 \\
\hline \hline TOTAL & 45 & 24728 & 100 \\
\hline
\end{tabular}

Source tableaux 1 et 2 : Recensement 1981, informations des autorités locales

3 Nos résultats sur ces trois langues sont donc représentatifs de la situation dans la région étudiée. Il est plus difficile d'estimer la représentativité des locuteurs du dialecte pontique, puisque ce groupe ne peut pas être facilement distingué des réfugiés ${ }^{2}$ grecs provenant d'autres parties de la Turquie. Néanmoins, nous avons visité 17 villages habités par des locuteurs de ce dialecte, ce qui nous a permis de nous faire une idée de la fréquence de son utilisation. Dans certains villages, les réfugiés d'Asie Mineure parlent aussi le turc (tableau 4).

Aspects socio-économiques

4 Le département de Florina a toujours été une zone agricole sous-développée, en partie à cause de conditions naturelles défavorables, et en partie pour des raisons sociales et historiques. Les conditions de pauvreté extrême qui ont marqué la région, depuis la période ottomane jusqu'aux années soixante, appartiennent désormais au passé. Toutefois il s'agit toujours d'une des régions les moins développées du pays. Il n'y a presque pas d'industrie: $53 \%$ de la population active est employée dans le secteur primaire contre $20 \%$ seulement dans le secondaire et $27 \%$ dans le tertiaire (les pourcentages au niveau national étant respectivement $25 \%, 27 \%$ et $47 \%{ }^{3}$ ). La centrale électrique DEI est l'employeur principal, mais une grande partie de la main-d'œuvre est 
recrutée dans d'autres régions de la Grèce. Il y a également douze industries de petite échelle qui emploient environ 500 travailleurs, mais la plupart d'entre eux sont recrutés uniquement sur contrats précaires à court terme. En agriculture, la propriété moyenne est de trois hectares, mais beaucoup en possède moins et il y a un nombre important de jeunes agriculteurs sans terre. Les principales productions agricoles sont les céréales, le tabac et l'élevage. L'agriculture a été modernisée et le niveau de vie amélioré, principalement grâce aux subventions de la CEE, mais le coût de production est élevé et la plupart des agriculteurs sont sous-employés pendant une grande partie de l'année. Le taux de chômage est parmi les plus élevés du pays ${ }^{4}$, tandis qu'un tiers seulement des chômeurs reçoit une indemnité de chômage ${ }^{5}$. Entre les groupes linguistiques il y a des différences marquées quant à l'accès aux emplois publics, à l'enseignement supérieur et à la propriété. Les refugiés sont les mieux lotis, tandis que les slavophones se trouvent, avec les Gitans, au plus bas de la hiérarchie sociale.

5 La région d'Aridea est plus prospère que celle de Florina. Son climat plus doux et sa plaine fertile permettent jusqu'à trois récoltes par an. La production agricole est largement commercialisée : les principales productions sont le tabac, les pêches et les cerises. Les cultures intensives pratiquées dans ce secteur de production compensent en partie le problème majeur de la région : le manque de terres. La propriété moyenne n'est que de 1,5-2 hectares. Depuis les années quatre-vingt, le niveau de vie des agriculteurs a été amélioré sensiblement grâce aux subventions de la CEE. Néanmoins, au cours des deux ou trois dernières années, la filière fruitière est entrée en crise, à la fois en raison de la surproduction et des problèmes de transport liés à la crise yougoslave. Actuellement, seuls 10 à $40 \%$ de la production de fruits sont commercialisés, le reste étant détruit. La région a connu une importante industrialisation, mais la plupart des usines sont petites et l'emploi fourni dépasse rarement quelques mois par an. Comme à Florina, presque toutes les usines sont entre les mains des réfugiés. Le taux de chômage parmi les jeunes ayant terminé leurs études secondaires est élevé, mais moins qu'à Florina. Il y a une différence importante entre villages pauvres et villages riches: la première catégorie inclut principalement des villages de montagne habités par des slavophones, tandis que les villages riches se trouvent dans la plaine et sont principalement habités par des réfugiés ${ }^{6}$. Cependant, dans ce département, les distinctions socio-économiques entre les groupes linguistiques sont moins nettes que dans celui de Florina.

La situation linguistique actuelle

6 Le grec est sans aucun doute la langue dominante dans toute la région, et les personnes qui ne le parlent pas du tout se comptent sur les doigts des deux mains. Cela s'explique par les facteurs de déclin examinés ci-dessous, ainsi que par l'absence totale de mesures en faveur des langues minoritaires. Il faut cependant souligner que malgré ce contexte peu favorable, elles ont fait preuve d'une grande vitalité. Le degré de préservation est plus élevé dans les villages relativement pauvres et isolés, mais les langues minoritaires sont également bien représentées dans certains villages qui sont parmi les plus dynamiques. Dans ces derniers, la langue minoritaire tend même à être la langue dominante dans la communication interpersonnelle.

7 Un fait plus étonnant concerne l'attitude de la population locale vis-à-vis des langues minoritaires. Les autorités grecques sont très sensibles à la question des langues minoritaires, parce que, à leur avis, une reconnaissance de ces langues ouvrirait la porte aux revendications territoriales des États voisins. Cependant, bien que cette vue 
officielle soit répercutée dans le discours local, notamment par l'intermédiaire des médias, l'existence et l'utilisation des langues minoritaires sont acceptées en règle générale comme une réalité indépendante de ses implications politiques éventuelles. Autre contradiction : les personnes d'un certain niveau d'éducation ont tendance à considérer l'utilisation des langues minoritaires comme un signe de "retard», mais beaucoup de locuteurs de telles langues sont fiers de leur patrimoine culturel, en particulier de leur langue. Nous avons constaté que ces sentiments de fierté se manifestaient surtout chez les Valaques et les Grecs pontiques. Il existe probablement aussi chez beaucoup de slavophones, mais ces derniers hésitent à exprimer publiquement de tels sentiments.

8 Une troisième remarque générale concerne l'usage des langues minoritaires par tranches d'âge. En règle générale, les personnes de plus de soixante ans parlent couramment la langue minoritaire comme première langue. Le groupe moyen, âgé entre trente et soixante ans, est bilingue, et, selon sa situation dans les différents villages, parle le grec ou la langue minoritaire comme première langue. Dans la famille, les parents parlent souvent la langue minoritaire entre eux, mais utilisent le grec pour s'adresser à leurs enfants, afin que ceux-ci puissent apprendre le grec correctement et améliorer leurs chances sur le marché du travail. Les grands-parents parlent souvent la langue minoritaire à leurs petits-enfants. Beaucoup d'adolescents en ont une bonne connaissance, mais, lorsqu'ils fréquentent les écoles mixtes en milieu urbain ou lorsqu'ils quittent leur village pour travailler ailleurs, ils tendent à la perdre. Les écoliers de l'enseignement primaire comprennent généralement la langue minoritaire, mais ne la parlent pas. Enfin, une remarque sur la différence entre les sexes : jusqu'à la génération précédente, les femmes étaient les principaux vecteurs des traditions familiales, y compris de la langue. En particulier, les grands-mères ont joué un rôle crucial dans la transmission de la langue ${ }^{7}$. Cela semble changer aujourd'hui : dans beaucoup de ménages, les femmes plus que les hommes tiennent à parler le grec à leurs enfants.

Facteurs de préservation et de déclin

9 Comme c'est le cas pour la plupart des minorités linguistiques, l'influence des institutions nationales dominées par la langue nationale est un facteur majeur de déclin pour les langues minoritaires. Ces institutions comprennent le système d'éducation, l'armée et les médias. L'abandon des langues minoritaires est aussi lié aux mariages mixtes, bien que, dans cette région, les mariages entre les groupes linguistiques soient un phénomène relativement récent et limité. Les effets de cette situation sont multipliés par un certain nombre de facteurs spécifiques à la région, liés souvent à la politique linguistique des autorités nationales et régionales. Le souvenir de la répression linguistique du passé (surtout sous le régime de Ioannis Metaxas, 1936-1940, et pendant la guerre civile), ainsi que le découragement actuel ont créé un climat de crainte en ce qui concerne l'utilisation des langues minoritaires. Cette dimension psychologique est plus marquée parmi les anciennes générations de slavophones, tandis qu'elle ne semble pas affecter les locuteurs du dialecte pontique. Un autre élément qui joue un rôle important parmi le groupe slavophone a été la création, dès les années cinquante, de crèches et d'écoles maternelles dans la plupart des villages slavophones, de sorte que les enfants apprennent le grec à un âge précoce. La génération actuelle de mères slavophones a fréquenté de telles écoles, ce qui pourrait expliquer que le rôle des femmes dans la transmission de la langue est moins important aujourd'hui que dans le passé. Enfin, on doit mentionner le rôle de 
l'émigration. Généralement, ceux qui ont émigré en Europe occidentale se sont intégrés dans une communauté de locuteurs grecs dans le pays d'accueil, et non pas dans un groupe de locuteurs de la langue minoritaire. Dans la mesure où beaucoup de ces émigrés sont revenus en Grèce ou visitent leur village régulièrement pendant les vacances, leur comportement linguistique a une certaine influence au niveau local.

Cependant, plusieurs phénomènes ont contribué à favoriser la préservation des langues minoritaires. En premier lieu il faut mentionner l'isolement relatif de la population rurale, l'ampleur limitée de l'industrialisation et le faible degré d'urbanisation (sauf pour le groupe des réfugiés). Ces facteurs empêchent la création d'un effet de « creuset "(melting-pot). Un deuxième élément est l'existence de divisions interethniques marquées dans la sphère socio-économique et le faible degré de mariage entre ces groupes. Troisièmement, la vitalité des traditions populaires, dans lesquelles la langue minoritaire joue souvent un rôle important (particulièrement pour les slavophones et les réfugiés pontiques). Outre ces facteurs internes, il y a également un certain nombre d'influences externes qui favorisent la sauvegarde des langues minoritaires. Évoquons d'abord le rôle des médias étrangers offrant des programmes dans la même langue ou une langue similaire aux langues minoritaires parlées dans la région. Les programmes qui peuvent être saisis dans la région sont ceux de la radio et de la télévision de Skopje et Tirana, et de la radio de Sofia. Ces programmes sont souvent enregistrés sur cassette ou vidéocassette et ensuite passés d'une famille à l'autre. Un autre élément important est les contacts avec des parents vivant à l'étranger dans un pays où la langue minoritaire est dominante et qui souvent ne comprennent pas le grec, puisqu'ils ont quitté leur village natal quand l'utilisation du grec était beaucoup plus limitée. Ce dernier facteur concerne surtout les anciens réfugiés politiques, qui ont fui la région après la guerre civile (1946-1949) et dont beaucoup sont des slavophones. Enfin, on devrait mentionner la présence de nouveaux immigrés et des "visites d'achats " d'étrangers en provenance de pays où les langues minoritaires sont dominantes, c'està-dire d'ex-Yougoslavie et d'Albanie. C'est pour le macédonien que l'influence de ces facteurs de préservation est la plus forte.

Degré de vitalité

11 Nous avons classé les villages en trois catégories, selon le degré de vitalité des langues minoritaires (tableaux 3 et 4 ) :

12 - dans le groupe 1, le niveau de préservation est le plus élevé. La langue minoritaire est la langue habituelle de communication, avec le grec, en public et en privé. Elle peut même être employée par les autorités communales dans leurs transactions avec les villageois ;

13 - dans le groupe 2, le pourcentage de personnes qui ont appris la langue minoritaire comme première langue est moins élevé que dans le groupe 1. La plupart des personnes de plus de trente ans ont appris les deux langues en même temps et sont bilingues, mais les personnes de plus de cinquante ou soixante ans se sentent plus à l'aise dans la langue minoritaire. Celle-ci est encore souvent utilisée dans le quotidien, mais plutôt dans le domaine privé qu'en public. En règle générale, les personnes de moins de vingt ans ne parlent pas la langue, mais ont un bon niveau de compréhension ;

14 - dans le groupe 3, la langue minoritaire est parlée uniquement par les personnes âgées et dans la majorité des cas uniquement en privé.

15 Dans les villes de Florina et d'Aridea on peut entendre toute la gamme des langues minoritaires, surtout pendant les jours de marché, quand les agriculteurs des villages 
descendent en ville pour vendre leurs produits. La langue habituelle de communication est évidemment le grec, puisque la population urbaine est en grande partie mixte. Ces deux villes sont aussi le lieu de résidence des Gitans, qui parlent, en dehors de leur propre langue (rom), toutes les autres langues en usage dans la région.

Usage des langues minoritaires

Tableau 3 - Département de Florina

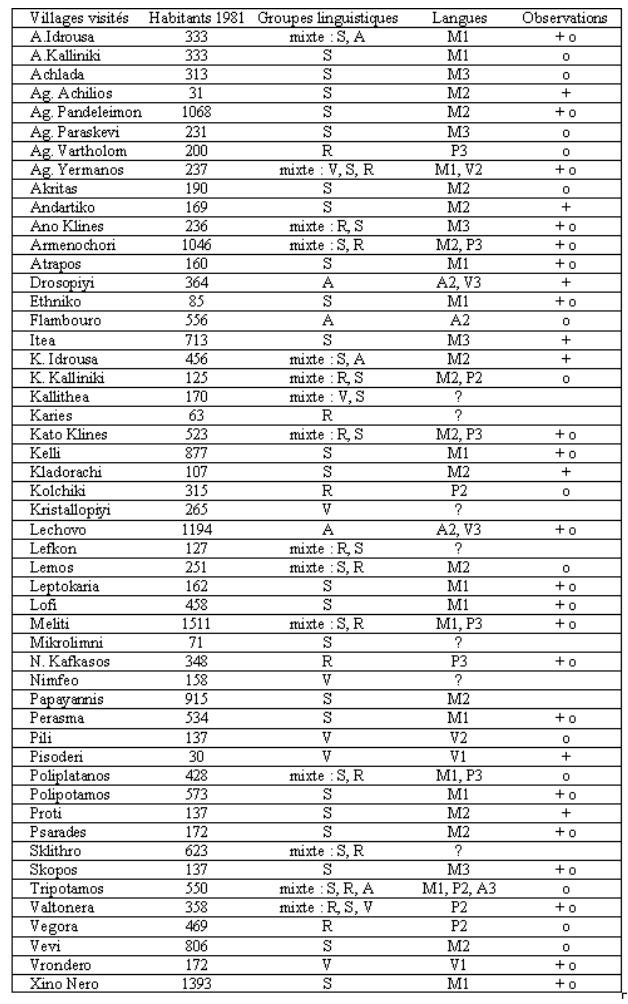




\section{Tableau 4 - Département d'Aridea}

\begin{tabular}{|c|c|c|c|c|}
\hline Villages visités & Habitants 1981 & Groupes linguistiques & Langues & Observations \\
\hline Apsalos & 1277 & Mixte $: \mathrm{R}, \mathrm{V}$ & $\mathrm{P} 2, \mathrm{~V} 2$ & 0 \\
\hline Archangelos & 709 & $\mathrm{~V}$ & V1 & +0 \\
\hline Chrisa & 1045 & Mixte : R, S & M2, T3 & +0 \\
\hline Filotia & 647 & Mixte : $\mathrm{S}, \mathrm{R}$ & M3 & 0 \\
\hline Foustani & 651 & Mixte : $\mathrm{R}, \mathrm{S}$ & M2, T3? & 0 \\
\hline Korifi & 126 & $\mathrm{~S}$ & M1 & + \\
\hline Likostomo & 382 & Mixte : S, R & $\mathrm{M} 2, \mathrm{P} 2, \mathrm{~T} 3$ & +0 \\
\hline Loutraki & 1066 & $\mathrm{~S}$ & M1 & +0 \\
\hline Megaplatanos & 382 & Mixte : R, S & M2, P2, T3? & 0 \\
\hline Milea & 787 & Mixte : R, S & M2, T2 & +0 \\
\hline Notia & 412 & Mixte : $\mathrm{R}, \mathrm{V}$ & $\mathrm{P} 2, \mathrm{~V} 2$ & +0 \\
\hline Orma & 685 & $\mathrm{~S}$ & M2 & +0 \\
\hline Periklia & 373 & $\mathrm{~V}$ & V1 & +0 \\
\hline Piperia & 481 & Mixte : R, S & $\mathrm{M} 2, \mathrm{P} 2, \mathrm{~T} 3$ & +0 \\
\hline Polikarpi & 1046 & $\mathrm{R}$ & $\mathrm{T} 2$ & +0 \\
\hline Promachi & 1754 & $\mathrm{~S}$ & M2 & 0 \\
\hline Sarakini & 356 & $\mathrm{~S}$ & M1 & +0 \\
\hline Sosandra & 1291 & Mixte :S, R & M2 & +0 \\
\hline Theodoraki & 805 & $\mathrm{~S}$ & M1 & 0 \\
\hline Xifiani & 923 & Mixte : $\mathrm{S}, \mathrm{R}$ & $\mathrm{M} 2 \mathrm{P} 2$ & 0 \\
\hline
\end{tabular}

Explication des symboles des tableaux 3 et 4

- Ad rubrique 3 (groupes linguistiques) : $\mathrm{S}=$ Slavophones

$\mathrm{R}=$ Refugiés

$\mathrm{V}=$ Valaques (Aroumains)

$A=$ Arvanites

Dans les villages mixtes les groupes sont mentionnés dans l'ordre de leur poids numérique.

- Ad rubrique 4 (langues) : $M$ = macédonien

$\mathrm{P}=$ dialecte pontique

$A=$ arvanitika

$\mathrm{V}=$ valaque (aroumain)

$\mathrm{T}=$ turc

1. La langue minoritaire est utilisée à tous les âges, en public et en privé. Elle a tendance a être la langue dominante dans les relations interpersonnelles. Connaissances très limitées du grec pour certaines personnes âgées.

2. La langue minoritaire est utilisée par des personnes de plus de 30 ans, en public et en privé. Les enfants ne l'utilisent pas habituellement, mais sont en mesure de la comprendre.

3. La langue minoritaire est utilisée uniquement par des personnes de plus de 60 ans, dans la plupart des cas seulement en privé.

- Ad rubrique 5 (observations) :

$+=$ observations de terrain

$\mathrm{o}=$ informations recueillies dans les villages, surtout auprès des autorités locales 


\section{NOTES}

1. Le rapport complet a été déposé auprès de la Commission européenne sous le titre Report to the European Commission. Minority Languages in Northern Greece. Study Visit to Florina and Aridea, september 1993.

2.1 Le terme « réfugié » est utilisé ici pour désigner les Grecs d'Asie Mineure qui se sont établis en Grèce dans les années vingt après l'échange de population entre la Turquie et la Grèce (Traité de Lausanne, 1924).

3. Eurostat, 1992, p. 129.

4. Selon le préfet du département à l'époque, Mme Peidou-Sidiropoulou, plus de $20 \%$ de la population active et $35 \%$ des jeunes était au chômage (Peidou-Sidiropoulou, Th. «Ferte kapno, molinsi kai fougara yia na doulepsei o kosmos » Oikonomikos Tachidromos, Édition spéciale sur la Grèce du Nord, 28.7.94, p. 89-90).

5. Information fournie par M. Neokaziotis, directeur de l'Agence pour l'emploi de Florina, 2.9.93.

6. Les informations sur l'économie locale proviennent d'un entretien avec M. Zisis Eleftheriadis, président du Conseil municipal d'Aridea, en novembre 1992.

7. Voir pour la région d'Aridea, G. Drettas, «Tant que les grand-mères parlent » Epitheorisi Kinonikon Erevnon, édition spéciale, 1981.

\section{RÉSUMÉS}

Ce document présente les principaux résultats d'une enquête de terrain effectuée en septembre 1993 et financée par la Commission européenne. L'objectif de cette enquête était d'analyser l'usage des langues minoritaires dans une région de la Grèce du Nord qui se présente comme une mosaïque linguistique. Dans cette région formée des départements de Florina et d'Aridea, en dehors de la langue dominante (le grec), sont encore en usage les langues suivantes: le macédonien, le valaque (proche du roumain), l'arvanitika (proche de l'albanais), le turc, le rom et le dialecte pontique (un dialecte grec utilisé par les anciens habitants de la mer Noire ou Pontos). L'équipe, composée de deux anthropologues néerlandaises, Riki Van Boeschoten et Helleen van der Minne, a visité en tout 72 des 139 villages de la région.

This document presents the results of a field survey carried out in September 1993 and funded by the European Commission. The aim was to analyse usage of minority languages in a region of Northern Greece, which resembles a linguistic mosaic. In this region made up of the departments of Florina and Aridea, aside from the dominant Greek language, the following languages are still in use: Macedonian, Valak (close to Romanian), Arvanitika (close to Albanian), Turkish, Rom and the Pontic dialect (a Greek dialect used by previous inhabitants of the Black Sea or Pontos). The team, made up of two Dutch anthropologists, Riki Van Boeschoten and Helleen van der Minne, visited 72 of the 139 villages in the region. 
AUTEUR

RIKI VAN BOESCHOTEN

Université de Thessalie 